\title{
Respeto al mínimo vital como un derecho humano ineludible en México
}

\author{
Respect for the vital minimum, as an inescapable human right in Mexico
}

\section{$O$ respeito pelo mínimo vital como um direito humano inescapável no \\ México}

\begin{abstract}
Gerardo Hernández Barrena
Centro de Estudios e Investigaciones para el Desarrollo Docente CENID A.C. México

barrenagerardo@yahoo.com.mx

https://orcid.org/0000-0003-1593-6402
\end{abstract}

\section{Resumen}

La Constitución Política de los Estados Unidos Mexicanos señala en su artículo 31, fracción IV, que todos los mexicanos están obligados a contribuir para el gasto público; sin embargo, antes de pagar, el Estado debe garantizar que el contribuyente subsanó sus necesidades de alimentación, vivienda, salud, educación, etc., para estar en condiciones de contribuir; esto recibe el nombre de mínimo vital, derecho humano que debe ser resguardado por parte de cualquier organismo gubernamental. Por eso, la presente investigación tuvo como objetivo dilucidar si en México el Estado protege el derecho al mínimo vital. Para ello, se ha trabajado tomando en cuenta los principios del método deductivo, pues se presenta el tema de lo general a lo particular. Además, se ha usado un instrumento tipo encuesta con la finalidad de conocer la opinión de los contribuyentes. Según los resultados conseguidos, se puede concluir que el Estado no considera para el pago de las contribuciones el derecho al mínimo vital de los contribuyentes, lo que violenta sus derechos humanos.

Palabras clave: contribuciones, derechos humanos, mínimo vital. 


\section{Revista Iberoamericana \\ de las Ciencias Sociales y \\ Humanísticas}

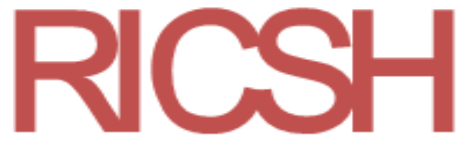

ISSN: 2395 - 7972

\section{Abstract}

The Constitution states in article 31 fraction IV that all Mexicans are required to contribute to public spending, however before paying, the State must ensure that the taxpayer met their needs for food, housing, health, education etc., in order to be able to contribute, this is named after the minimum vital, human right to be protected by any governmental body; that is why this research aims to clarify whether the State protects the right to the minimum of life, which will be carried out under the deductive method, presenting the subject of the general to the particular, also using a survey-type instrument with the purpose to obtain data that determine the opinion of taxpayers, concluding that the State does not consider for the payment of contributions the right to the vital minimum of taxpayers, then violating their human rights.

Keywords: contribution, human rights, minimum vital.

\section{Resumo}

A Constituição Política dos Estados Unidos Mexicanos indica em seu artigo 31, inciso IV, que todos os mexicanos são obrigados a contribuir para os gastos públicos; Porém, antes de efetuar o pagamento, o Estado deve garantir que o contribuinte tenha atendido às suas necessidades de alimentação, moradia, saúde, educação, etc., para poder contribuir; Isso é chamado de mínimo vital, um direito humano que deve ser protegido por qualquer órgão governamental. Portanto, a presente investigação teve como objetivo elucidar se no México o Estado protege o direito ao mínimo vital. Para isso, trabalhamos levando em consideração os princípios do método dedutivo, uma vez que o assunto é apresentado do geral ao particular. Além disso, um instrumento do tipo survey tem sido utilizado para conhecer a opinião dos contribuintes. De acordo com os resultados obtidos, pode-se concluir que o Estado não considera o direito dos contribuintes a um mínimo vital para o pagamento das contribuições, o que viola seus direitos humanos.

Palavras-chave: contribuições, direitos humanos, mínimo vital.

Fecha Recepción: Febrero 2020

Fecha Aceptación: Diciembre 2020 


\section{Revista Iberoamericana \\ de las Ciencias Sociales y \\ Humanísticas}

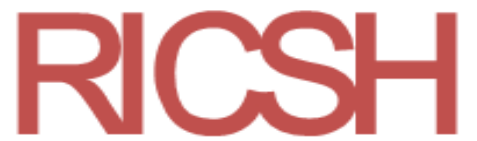

ISSN: $2395-7972$

\section{Introducción}

Los derechos humanos definen las relaciones entre los individuos y las estructuras de poder; estos delimitan el poder del Estado y, al mismo tiempo, le exigen a dicho ente que adopte medidas para garantizar condiciones en las que todas las personas puedan disfrutar de sus derechos humanos (Unión Interparlamentaria, 2016). Los derechos económicos, sociales y culturales nacen de la dignidad humana y son, por ende, inherentes a la persona humana, por lo que están directamente relacionados con la protección de las necesidades y capacidades que garantizan una calidad de vida (Nowak, 2005).

Uno de los pilares básicos del estado de derecho es la garantía de la libertad, pero para que los individuos puedan disfrutarla realmente es preciso que dispongan de un mínimo de seguridad económica. La Constitución Política de los Estados Unidos Mexicanos establece principios tributarios — también llamados principios constitucionales-, entre los que se destacan los de justicia, seguridad jurídica, legalidad, proporcionalidad y equidad. Del principio de proporcionalidad nace el derecho al mínimo vital, que procura asegurar la capacidad económica para que el contribuyente cubra sus necesidades primarias y las de su familia. En ese contexto, el principio de equidad radica en la igualdad ante la ley tributaria, que sirve para determinar el pago del tributo de acuerdo con la capacidad económica (Ruiz López, 2015).

Se puede decir, entonces, que el mínimo vital es un derecho por cuya virtud las personas no pueden ser llamadas a contribuir si no satisfacen sus necesidades básicas, teniendo en cuenta los principios de proporcionalidad y equidad (Hernández, 2016). En tal sentido, el artículo 31 fracción IV de la Constitución Política de los Estados Unidos Mexicanos establece que es obligación del mexicano contribuir con el gasto público de manera proporcional y equitativa según dispongan las leyes; sin embargo, para que el contribuyente pueda cumplir con esta norma constitucional debería, en primera instancia, satisfacer sus necesidades elementales, así como las de su familia.

\section{Planteamiento del problema}

En la actualidad la protección de los derechos humanos juegan un papel fundamental para las personas, de ahí que ahora más que nunca los ciudadanos exijan al Estado el amparo más amplio por ser el principal encargado de promover, respetar, proteger y garantizar dichos derechos; en materia tributaria, la protección debe abarcar el aspecto económico de los 


\section{Revista Iberoamericana \\ de las Ciencias Sociales y \\ Humanísticas}

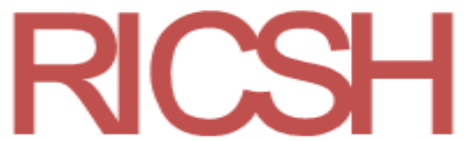

ISSN: 2395 - 7972

contribuyentes. Es decir, para que las personas paguen sus contribuciones, primero deben subsanar sus necesidades de alimentación, vivienda, salud y educación; a ello se le conoce como protección al mínimo vital de los contribuyentes, concepto que no es respetado por la autoridades fiscales en el ámbito contributivo.

\section{Objetivos}

\section{Objetivo general}

- $\quad$ Evidenciar la violación al mínimo vital de los contribuyentes, lo cual sucede desde el momento en que las autoridades fiscales no se cercioran de si antes de contribuir, dicho contribuyente ha subsanado sus necesidades de alimentación, vivienda, salud y educación.

\section{Objetivos específicos}

- Conocer el origen de los derechos humanos en el ámbito internacional.

- $\quad$ Examinar la protección de los derechos humanos en México.

- Dilucidar sobre la protección y respeto al mínimo vital de los contribuyentes por parte de las autoridades fiscales.

\section{Desarrollo}

\section{El mínimo vital}

Desde su nacimiento toda persona tiene derechos naturales derivados de la naturaleza y la razón humana. En el ámbito internacional, el 10 de diciembre de 1948 fueron reconocidos 30 artículos en la Declaración Universal de los Derechos Humanos, en donde fue plasmado que todos los seres humanos nacen libres e iguales en dignidad y derechos, y dotados como están de razón y conciencia y deben comportarse fraternalmente los unos con los otros. Por ello, se han fijado claramente los derechos y las libertades a los que todo ser humano puede aspirar de manera inalienable y en condiciones de igualdad (Naciones Unidas, 2015).

México, por supuesto, no fue ajeno a esta declaración, ya que el 10 de junio de 2011 a través de norma constitucional se dejó atrás el concepto de garantías individuales para dar paso a los derechos humanos y sus garantías, que quedó registrado en el título primero, capítulo 1, de la Constitución Política de los Estados Unidos Mexicanos publicada en el Diario Oficial de la Federación. 


\section{Revista Iberoamericana \\ de las Ciencias Sociales y \\ Humanísticas}

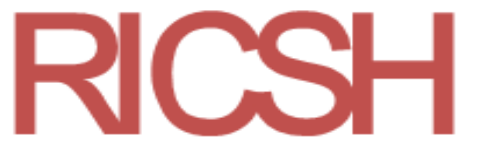

ISSN: 2395 - 7972

Ahora bien, el mínimo vital es un derecho humano fundamental, ya que se refiere a los ingresos que tiene una persona para financiar sus necesidades básicas y para poder hacer cumplir el derecho de la dignidad humana. En tal sentido, uno de los derechos de toda persona que trabaja es su salario, el cual debe permitir cubrir sus necesidades no solo para sí misma, sino también para su familia, por lo que se relaciona directamente con el goce y la satisfacción de los derechos humanos.

En la edad antigua, en Mesopotamia el salario de los trabajadores correspondía a la asignación de una unidad de trigo por jornal, mientras que en Grecia y en Roma había trabajadores esclavos y libres que recibían comida, vestido, calzado y, en ocasiones, una pequeña porción de tierra. Sería hasta el año 300 —reinado por Cayo Aurelio Valerio Diocleciano - cuando se fijó una lista real de salarios para todos los trabajadores.

Luego, en la Edad Media abundaban ordenanzas que fijaban los salarios; de hecho, en 1349 y 1350 se amenazaba con prisión a quien exigiera un salario más elevado que el recibido 20 años atrás. A partir de 1350, antes de la peste, los salarios habían permanecido estáticos; después de ello solo aumentaron $4 \%$, y fue hasta 1375 que se registró en un $14 \%$. En la época moderna, la llegada de la revolución industrial (que significó la aplicación de máquinas por mano de obra) ocasionó el desplazamiento del hombre con salarios más bajos que no bastaban para satisfacer lo mínimo en la vida del trabajador y de su familia (Juárez, 2013).

Luego en 1839, la ley de los pobres se enfocó en conceder el mínimo trigo por semana para procurar que los campesinos empobrecidos no se revelaran. Después, en el siglo XX Inglaterra reivindicó la creación de un dividendo y en Francia se propugnó un mínimo social garantizado para todo individuo desde el nacimiento hasta la muerte. Posteriormente, el 10 de diciembre de 1948, con la Declaración Universal de los Derechos Humanos, fue aprobado un doble reconocimiento: por una parte, el denominado mínimo vital o derecho del trabajador a "una remuneración equitativa y satisfactoria, que le asegure, así como a su familia, una existencia conforme a la dignidad humana y que será completada, en caso necesario, por cualesquiera otros medios de protección social" (artículo 23.3); por otra parte, el derecho de toda persona a "un nivel de vida adecuado que le asegure, así como a su familia, la salud y el bienestar y, en especial, la alimentación, el vestido, la asistencia médica y los servicios sociales necesarios" (artículo 25.1) (Senado de la República, 21 de junio de 2017).

El Pacto Internacional de Derechos Económicos, Sociales y Culturales — aprobado por la Asamblea General de Naciones Unidas en 1966, y cuya entrada en vigor se produjo en 


\section{Revista Iberoamericana \\ de las Ciencias Sociales y \\ Humanísticas}

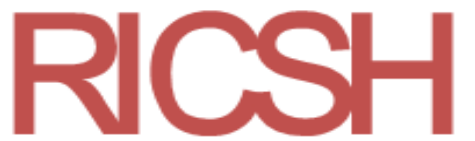

ISSN: 2395 - 7972

1976 - contiene también este doble reconocimiento del derecho a un mínimo vital. En el artículo 7 se establece el derecho de todos los trabajadores a una remuneración suficiente para proporcionarles a ellos y a sus familias unas condiciones de existencias dignas. Asimismo, en el artículo 11 se reconoce "el derecho de toda persona a un nivel de vida adecuado para sí y su familia, incluso alimentación, vestido y vivienda adecuados y a una mejora continua de las condiciones de existencia” (Rosales, 2017).

Por otra parte, la obra Salario mínimo y derechos humanos (Comisión Nacional de los Derechos Humanos [CNDH], 2016) señala que en 1980 en Nueva Zelanda y Australia se adoptó el concepto salario o ingreso mínimo, ya que la remuneración para los trabajadores —especialmente para mujeres y jóvenes - era tan baja que no les era suficiente para sobrevivir ellos y sus familias. Este concepto fue adoptado en varios países. Por ejemplo, en Colombia se decretó que el mínimo vital es un derecho innominado, construido a partir de la interpretación de la Constitución que se originó en un concepto similar: mínimo existencial. Igualmente, en Costa Rica — de acuerdo con la segunda sala de la Corte Suprema de Justicia - se estableció que este puede ser superado por voluntad de las partes, por lo que el monto salarial permitirá cubrir las necesidades en orden material, moral y cultural. Guatemala, por otra parte, reconoció el derecho al mínimo vital asegurando un salario mínimo para cubrir las necesidades básicas del trabajador.

En 1962, México incorporó la figura de salario mínimo al artículo 123 de la Constitución Política de los Estados Unidos Mexicanos en su apartado A, fracción VI, refiriendo un monto irreducible que debe recibir por mandato jurídico toda persona que realiza un trabajo para cubrir sus necesidades básicas y las de su familia. Es importante señalar que nuestro país fue uno de los primeros en establecer la figura de salario en su Constitución Política, iniciativa presentada por el Lic. Adolfo López Mateos, entonces presidente de la República. De hecho, este propósito se materializó en el artículo 90 de la Ley Federal del Trabajo (1970), donde en su segundo párrafo se fija lo siguiente: "El salario mínimo deberá ser suficiente para satisfacer las necesidades normales de un jefe de familia en el orden material, social y cultural, y para proveer a la educación obligatoria de los hijos".

Asimismo, en el artículo 31, fracción IV, de nuestra Constitución (Constitución Politíca de los Estados Unidos Mexicanos, 1917) se determinó como una obligación que los mexicanos debían contribuir para los gastos públicos de manera proporcional y equitativa, lo cual dio origen a los cuatro principios fundamentales de gasto público, proporcionalidad, equidad y 


\section{Revista Iberoamericana \\ de las Ciencias Sociales y \\ Humanísticas}

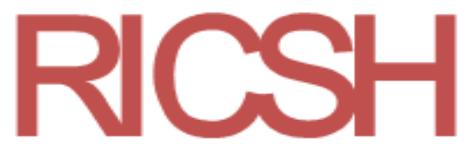

ISSN: $2395-7972$

legalidad.

Del principio de proporcionalidad nace el derecho al principio del mínimo vital, que intenta asegurar la capacidad económica para que el contribuyente cubra sus necesidades primarias y las de su familia (Hernández, 2016). El mínimo vital, como proyección del principio de proporcionalidad tributaria, constriñe al legislador tributario para que al momento de diseñar el objeto de las contribuciones e identificar la capacidad idónea para contribuir, respete un umbral libre o aminorado de tributación, correspondiente a los recursos necesarios para la subsistencia de las personas, en donde le está vedado introducirse por no estar legitimada constitucionalmente la imposición de gravámenes sobre ese mínimo indispensable (Tenorio, 2013). Para otros autores como Benvenuto (1959), el "mínimo para la existencia" no representa una capacidad contributiva, sino el costo de vivir, que no puede ser disminuido por los impuestos, pues se estaría transfiriendo el impuesto sobre otros contribuyentes.

Ahora bien, resumiendo diversas sentencias del Tribunal Constitucional Alemán, el derecho fundamental al mínimo vital deriva de los principios de estado social de derecho, dignidad humana y solidaridad, en concordancia con los derechos fundamentales a la vida, a la integridad personal y a la igualdad; tiene por objeto evitar que una persona se vea reducida en su valor intrínseco como ser humano debido a que no cuenta con las condiciones materiales que le permitan llevar una existencia digna. Este derecho busca garantizar que la persona, centro del ordenamiento jurídico, no se convierta en instrumento de otros fines, objetivos, propósitos, bienes o intereses, por importantes o valiosos que ellos sean. Es decir, se busca proteger a la persona contra toda forma de degradación que comprometa su valor intrínseco, concretamente en lo que se refiere a las condiciones materiales básicas e indispensables para asegurar una supervivencia digna y autónoma, por lo que constituye un límite al poder impositivo del Estado (Gerencie.com, 2019).

Se podría argumentar que las autoridades fiscales protegen el mínimo vital de los contribuyentes al permitir, por ejemplo, que las personas que perciban un salario mínimo no serán sujetas de retención de impuesto, o bien a la mayoría de los demás contribuyentes les permite disminuir de sus ingresos gastos o deducciones relacionadas con su actividad para con ello disminuir su carga tributaria; sin embargo, en ese contexto la Suprema Corte de Justicia de la Nación (Tesis: P. VI/2013 novena época 159821 pleno libro 1, diciembre de 2013, tomo I, pág. 135 tesis aislada) ha señalado que el mínimo vital no es exclusivo de la clase trabajadora, aunque para ellos es importante dejarles a salvo el salario mínimo. No 


\section{Revista Iberoamericana \\ de las Ciencias Sociales y \\ Humanísticas}

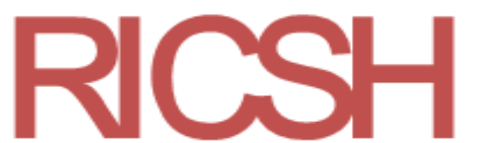

ISSN: 2395 - 7972

obstante, también señala que "el derecho al mínimo vital no es una prerrogativa exclusiva de la clase trabajadora, ni su contenido se agota al exceptuar de embargo, compensación o descuento al salario mínimo"; a contrario sentido quiere decir que cualquier persona debe ser sujeta a un mínimo vital sin tomar en cuenta su actividad.

Al respecto, se han realizado estudios recientes en donde se demuestra que en promedio una familia con 4.15 integrantes tiene un gasto mensual de $\$ 30013.00$; por tanto, y de acuerdo con un análisis de Coneval, para que una familia con un promedio de 4 integrantes no se encuentre en estado de pobreza debe de tener un ingreso mensual de $\$ 12458.76$ (Rodríguez y Contreras, 2018), aunque con ello cualquiera de las cantidades expresadas rebasa por mucho el salario mínimo, por lo cual es sujeto de impuesto la cantidad con la que una familia debe cubrir sus necesidades.

Algo similar sucede con las deducciones autorizadas, ya que solamente los contribuyentes pueden disminuir su carga tributaria si los gastos son relacionados con su actividad; por tanto, resulta indubitable que al no poder disminuir conceptos como alimentación, salud y vivienda, no se está protegiendo el mínimo vital de los contribuyentes.

\section{Metodología, técnicas y materiales empleados}

La presente investigación fue de tipo correlacional, ya que se indagaron las causas y efectos de la protección del mínimo vital de los contribuyentes. Se aplicó el método deductivo porque se analizaron los derechos humanos y el mínimo vital, de lo general a lo particular. El tipo de estudio fue documental y su enfoque fue, por una parte, cualitativo porque se efectuó un análisis de fuentes secundarias como libros, periódicos, revistas y páginas de internet, y, por otra parte, cuantitativo porque se presentaron en porcentajes y cifras los resultados de encuestas realizadas a empresarios y profesionistas.

Para el estudio se consideró una muestra de 50 empresarios y contadores de una población de 100 personas, los cuales fueron seleccionados de manera aleatoria, empleando una prueba piloto y realizando la corrección del instrumento para filtrar a los encuestados que tuvieran alguna experiencia en el tema. El instrumento demostró su fiabilidad porque se hallaron resultados similares en la aplicación de la prueba piloto y en la obtención de datos definitivos. Los ítems fueron validados con ayuda de expertos en el tema. 


\section{Revista Iberoamericana \\ de las Ciencias Sociales y Humanísticas}

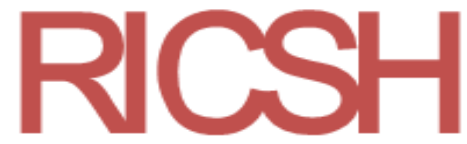

\section{Resultados}

Con la finalidad de establecer un conocimiento general y dar un soporte con enfoque contributivo a la protección del mínimo vital y a los derechos humanos, se aplicó un cuestionario a 50 empresarios y contadores. Los resultados se muestran en las siguientes figuras.

Figura 1. ¿Sabe qué son los derechos humanos?

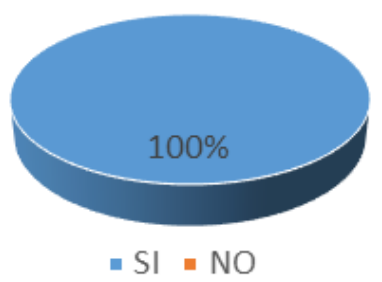

Fuente: Elaboración propia

En la figura 1 se observa que $100 \%$ de los entrevistados conoce qué son los derechos humanos, lo que resulta relevante, ya que cuanto más se conozca del tema más protección se puede exigir al Estado.

Figura 2. ¿Sabe que la Constitución Política de los Estados Unidos Mexicanos protege los derechos humanos?

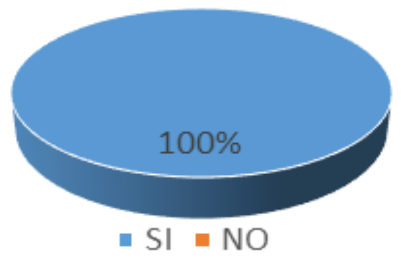

Fuente: Elaboración propia

La figura 2 enseña que todos los entrevistados saben que nuestra Constitución protege los derechos humanos, lo que refleja indubitablemente que las reformas constitucionales en esta materia no han pasado inadvertidas para los ciudadanos. 


\section{Revista Iberoamericana \\ de las Ciencias Sociales y \\ Humanísticas}

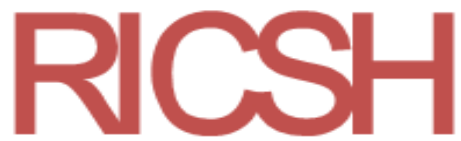

ISSN: $2395-7972$

Figura 3. ¿Conoce qué organismos internacionales protegen sus derechos humanos?

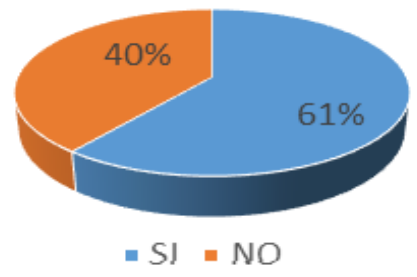

Fuente: Elaboración propia

La figura 3 enseña que $61 \%$ de la población tiene conocimiento de los organismos internacionales que protegen sus derechos humanos, lo que indica que se requiere mayor difusión sobre este aspecto con la finalidad de que los ciudadanos conozcan las diversas instituciones internacionales y su función específica.

Figura 4. ¿Conoce qué organismos nacionales protegen sus derechos humanos?

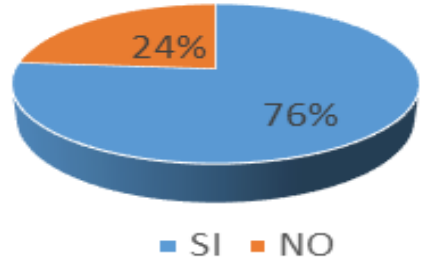

Fuente: Elaboración propia

De acuerdo con la figura 4, 76 \% de los entrevistados conocen los organismos nacionales que protegen sus derechos humanos; esta cifra resulta un tanto contradictoria, pues si bien en la figura 1 todos sabían qué eran los derechos humanos, el $24 \%$ no conoce las instituciones del país que se encargan de defenderlos.

Figura 5. ¿Sabe lo que es el mínimo vital?

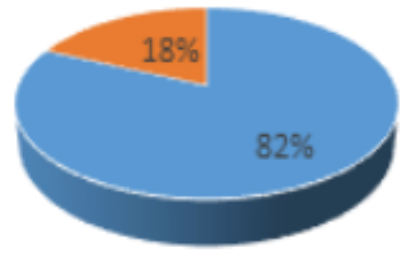

$=\mathrm{SI}=\mathrm{NO}$

Fuente: Elaboración propia 


\section{Revista Iberoamericana \\ de las Ciencias Sociales y \\ Humanísticas}

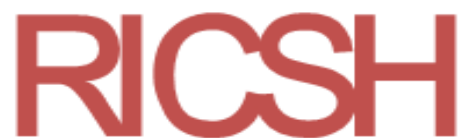

ISSN: 2395 - 7972

En la figura 5 se observa que $82 \%$ conoce qué es el mínimo vital, lo que demuestra que no todos saben este precepto, de ahí que pueda no ser exigido por los contribuyentes, a pesar de que se encuentre protegido por la propia Constitución y sea considerado como un derecho humano.

Figura 6. ¿Considera que al no ser sujetos del impuesto sobre la renta los trabajadores que perciben salario mínimo, se está protegiendo su mínimo vital?

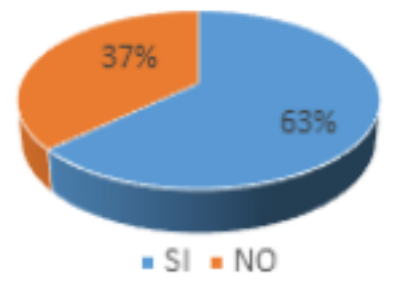

Fuente: Elaboración propia

En la figura 6 se muestra que $63 \%$ de la población considera que al no cobrar el impuesto sobre la renta a los trabajadores que perciben el salario mínimo, se está protegiendo su mínimo vital; no obstante, como se ha demostrado, dicho salario no subsana las necesidades de una familia promedio, lo que sin lugar a duda compromete la alimentación, vivienda y educación de los ciudadanos.

Figura 7. ¿Considera que al permitir a ciertos contribuyentes que disminuyan de sus ingresos gastos o deducciones, se está protegiendo su mínimo vital?

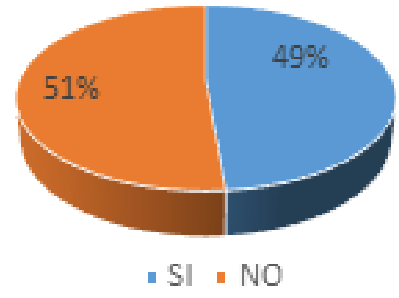

Fuente: Elaboración propia

En la figura 7 se aprecia que $51 \%$ de los entrevistados considera que no se protege el mínimo vital al permitir a ciertos contribuyentes que disminuyan de sus ingresos gastos o deducciones. Al respecto, se debe señalar que el mínimo vital es un principio que va más allá de disminuir una erogación de los ingresos, por lo que se debe reconocer que el contribuyente debe subsanar de su ganancia gastos relacionados con su subsistencia y la de su familia, aunque dichos gastos no siempre son reconocidos $\longrightarrow$ o no totalmente - para el pago de sus 


\section{Revista Iberoamericana \\ de las Ciencias Sociales y \\ Humanísticas}

contribuciones.

Figura 8. ¿Considera de manera general que las autoridades fiscales respetan y protegen su mínimo vital?

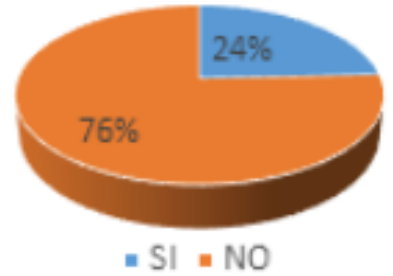

Fuente: Elaboración propia

En la figura 8 se nota que $76 \%$ de los encuestados considera que las autoridades no respetan ni protegen el mínimo vital. Sin lugar a duda, la palabra del ciudadano y su percepción sobre la protección de su mínimo vital resultan trascendentes para determinar esta falta a los derechos humanos, lo cual constitucionalmente se transforma en un quebrantamiento a la ley suprema de nuestro país.

\section{Discusión}

Para realizar un estudio relacionado con el mínimo vital se encuentran limitaciones derivadas de la falta de conocimiento acerca del concepto. Por ello, se puede asegurar que aunque es cierto que este derecho se encuentra protegido por la Constitución Política de los Estados Unidos Mexicanos, en la práctica no se puede exigir su cumplimiento cuando se desconoce su alcance.

En ese sentido, diversos autores han dado su concepto del mínimo vital, y al respecto concluyen que antes de contribuir el sujeto debe subsanar sus necesidades básicas de alimentación y vivienda, entre otras. De hecho, para García Bueno (2002) la capacidad contributiva está condicionada por una exigencia lógica: la presencia de capacidad económica, ya que el hecho de que el sujeto cuente con un índice de capacidad económica no garantiza el nacimiento de su capacidad contributiva. Carmona Cuenca (2006) también indica que cuando hablamos del mínimo vital debemos referirnos al derecho de todos los individuos a contar con una cantidad mínima para hacer frente a sus necesidades más básicas. Por otro lado, se ve reflejado un lazo muy débil entre el derecho humano y el aspecto fiscal, ya que a pesar de estar reconocido como un derecho tributario, la satisfacción de las necesidades básicas queda en duda al no corroborarse este hecho por parte de la autoridad, 


\section{Revista Iberoamericana \\ de las Ciencias Sociales y \\ Humanísticas}

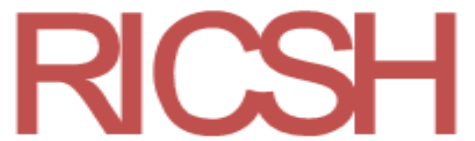

ISSN: $2395-7972$

lo que deja a la deriva a los derechos humanos en materia fiscal.

Sin embargo, con el paso de los años se le ha dado un mayor énfasis a la protección de los derechos humanos por parte de distintas autoridades, dependencias y organizaciones, donde indudablemente se incluye el aspecto tributario, por lo que de cierta manera dará pauta al debido cumplimiento del mínimo vital.

\section{Conclusiones y recomendaciones}

En la actualidad los derechos humanos juegan un papel primordial en la protección de las personas en aspectos sociales, políticos, de género, de medio ambiente y económicos, por lo que la preocupación a nivel internacional ha permitido crear diversos organismos y normas que resguardan la dignidad de las personas. En ese sentido, el ámbito económico debe abarcar la esfera contributiva de los ciudadanos y la interpretación de las leyes deben favorecer en todo momento a los sujetos pasivos en beneficio del principio pro-persona.

En ese aspecto, México no ha sido la excepción, ya que con las reformas constitucionales que entraron en vigor el 11 de junio del 2011 se percibe y se señala que todas las normas relativas a los derechos humanos se interpretarán de conformidad con la Constitución y con los tratados internacionales para favorecer en todo tiempo a las personas. Asimismo, en el artículo $1 .^{\circ}$ de nuestra Constitución, tercer párrafo, se indica que las autoridades tienen la obligación de promover, respetar, proteger y garantizar los derechos humanos, lo que indudablemente incluye la protección al derecho al mínimo vital, por lo que la autoridad debe cerciorarse de que el contribuyente ha subsanado todas sus necesidades básicas, así como las de su familia, para estar en condiciones de poder pagar sus contribuciones.

Desde nuestro punto de vista, es evidente que la autoridad está omitiendo un paso importante en el proceso de recaudación de contribuciones: verificar que el contribuyente está en condiciones aptas para cumplir con su tributo. En otras palabras, como lo establece el artículo 123 constitucional, fracción VI, se debe constatar que el ingreso que se percibe por el trabajo o servicio prestado es suficiente para cubrir sus necesidades básicas, así como las de su familia, pues - en caso contrario - el contribuyente no estaría obligado al pago de impuestos.

Es importante señalar que el estudio que se realiza por parte de la autoridad a las familias debe ser periódico para conocer las necesidades en orden material, social y cultural, ya que de esta manera se pueden crear iniciativas para mejorar el poder adquisitivo, lo que les 


\section{Revista Iberoamericana \\ de las Ciencias Sociales y \\ Humanísticas}

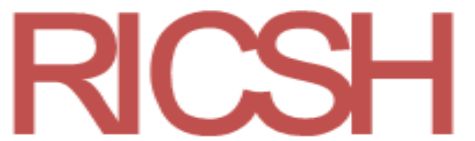

ISSN: $2395-7972$

permitirá a las personas el goce y disfrute de sus derechos humanos.

Si el Estado o nuestras autoridades hacendarias protegieran el mínimo vital, repercutiría de manera positiva tanto en los contribuyentes como en las autoridades, ya que habría una evolución en el sistema recaudatorio y habría un mejor reconocimiento de los derechos humanos y del mínimo vital de los sujetos obligados a contribuir.

En definitiva, se abren caminos para nuevas líneas de investigación relacionadas con la protección de los derechos humanos en materia fiscal, ya que el ámbito tributario abarca más actos y procedimientos donde las autoridades hacendarias deben garantizar, proteger e interpretar las leyes en beneficio del principio pro-persona.

\section{Referencias}

Benvenuto, G. (1959). Principios de ciencia de las finanzas (6. ${ }^{\mathrm{a}}$ ed.). Buenos Aires: Depalma.

Carmona Cuenca, E. (2006). Los derechos sociales de prestación y el derecho a un mínimo vital. Nuevas Políticas Públicas: Anuario multidisciplinar para la modernización de las Administraciones Públicas, (2), 172-197.

Comisión Nacional de los Derechos Humanos [CNDH] (2016). Salario mínimo y derechos

humanos. Recuperado de http://www.cndh.org.mx/sites/all/doc/OtrosDocumentos/Doc_2016_018.pdf

Constitución Politíca de los Estados Unidos Mexicanos (1917). México: Cámara de Diputados del H. Congreso de la Unión. Última reforma publicada DOF 06-03-2020.

García Bueno, M. C. (2002). El principio de capacidad contributiva como criterio esencial para una reforma fiscal. México: Universidad Nacional Autónoma de México.

Gerencie.com. (2019). El mínimo vital y el salario mínimo. Recuperado de http://www.gerencie.com/derecho-fundamental-al-minimo-vital.html

Hernández, B. G. (2016). El mínimo vital y los impuestos sobre la renta y al valor agregado. México: Cajica.

Juárez, R. A. (2013). Administracion de la compensación. México. Recuperado de https://cucjonline.com/biblioteca/files/original/8b6446e4bc766d7b63f03b4170474e 49.pdf

Ley Federal del Trabajo (1970). Mexico: Cámara de Diputados del H. Congreso de la Unión. Última reforma publicada en el DOF 02-07-2019. 


\section{Revista Iberoamericana \\ de las Ciencias Sociales y \\ Humanísticas}

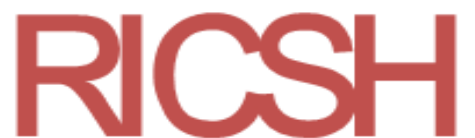

ISSN: 2395 - 7972

Naciones Unidas (2015). Declaracion Universal de los Derechos Humanos. Recuperado de https://www.un.org/es/documents/udhr/UDHR_booklet_SP_web.pdf

Nowak, M. (2005). Derechos humanos: manual para parlamentarios. Recuperado de https://www.ohchr.org/Documents/Publications/training13Newsp.pdf

Rodríguez, Á. G. y Contreras, P. T. (2018). El mínimo vital en México. Universidad Nacional Autónoma de México.

Rosales, C. M. (2017). El mínimo vital como institución de justicia elemental. Revista IUS Doctrina, $\quad$ 10(10). Recuperado de https://revistas.ucr.ac.cr/index.php/iusdoctrina/article/view/31912

Ruiz López, M. E. (2015). Puntos fundamentales de los principios de proporcionalidad y equidad tributaria. Ruiz Consultores. Recuperado de https://www.ruizconsultores.com.mx/que-son-los-principios-de-proporcionalidad-yequidad-tributaria/

Senado de la República (21 de junio de 2017). Iniciativas de ciudadanos legisladores. Gaceta de la Comisión Permanente. Recuperado de https://www.senado.gob.mx/64/gaceta_comision_permanente/documento/72549

Tenorio, I. C. (2013). Conceptos constitucionales de la contribución o del derecho al mínimo vital. Recuperado de http://cesmdfa.tfja.gob.mx/investigaciones/historico/pdf/conceptosconstitucionales. pdf

Unión Interparlamentaria (2016). Derechos Humanos. Recuperado de https://www.ohchr.org/Documents/Publications/HandbookParliamentarians_SP.pdf 